\title{
Management of the neonate at risk for early- onset Group B streptococcal disease (GBS EOD): new paediatric guidelines in Belgium
}

\author{
L. Mahieu ${ }^{1}$, J.-P. Langhendries ${ }^{2}$, V. Cossey ${ }^{3}$, C. De Praeter ${ }^{4}$, P. Lepage ${ }^{5}$, \\ P. Melin 6
}

\begin{abstract}
${ }^{1}$ Division Neonatology, Antwerp University Hospital, Antwerp University, Department of Paediatrics, Belgium, ${ }^{2}$ CHC-Site St Vincent, Neonatal Intensive Care Unit, Liège-Rocourt, Belgium, ${ }^{3}$ Neonatal Intensive Care Unit, University Hospitals Leuven, Belgium, ${ }^{4}$ Department of Neonatology, Ghent University Hospital, Belgium, ${ }^{5}$ Department of Paediatrics, Université Libre de Bruxelles, Hôpital Universitaire des Enfants Reine Fabiola, Belgium, ${ }^{6}$ Medical Microbiology Department, National Reference Centre for Streptococcus agalactiae, University Hospital of Liège, Belgium
\end{abstract}

\begin{abstract}
Despite group B streptococcal (GBS) screening in late pregnancy and intrapartum antimicrobial prophylaxis, early-onset sepsis in neonates remains a common source of neonatal morbidity and mortality especially in preterm neonates. The identification of neonates with early-onset sepsis is usually based on perinatal risk factors. Clinical signs are aspecific and laboratory tests are not sensitive. Therefore, many clinicians will overtreat at-risk infants. Inappropriate treatment with antibiotics increases the risk for lateonset sepsis, necrotizing enterocolitis, mortality, and prolongs hospitalisation and costs. In 2003, the Belgian Health Council published guidelines for the prevention of perinatal GBS infections. This report presents the Belgian paediatric management guidelines, which have been endorsed by the Belgian and Flemish societies of neonatology and paediatrics. The most imported changes in the 2014 guidelines are the following: recommendations for a lumbar puncture; clarification of normal spinal fluid parameters and blood neutrophil indices corrected for gestation age; specific timing for diagnostic testing after birth; no indication for diagnostic testing in asymptomatic newborns unless additional risk factors; a revised algorithm for management of neonates according to maternal and neonatal risk factors; and premature infants described as those below 35 weeks instead of 37 weeks. The guidelines were made on the basis of the best evidence and on expert opinion when inadequate evidence exists.
\end{abstract}

Keywords: GBS, Belgium, Guideline, Paediatric, Management, Neonate

\section{Introduction}

Since the seventies, the incidence of neonatal group B streptococcal sepsis and meningitis has increased dramatically in all industrialized countries. ${ }^{1}$ Today, group B streptococcal (GBS) is identified as the leading cause of invasive bacterial infections in neonates. The reported attack rates for the earlyonset disease (EOD) (birth to age 7 days) range from 0.5 to 4 cases per 1000 live births. ${ }^{2-4}$ In the late-onset form occurring in infants aged $>1$ week, the attack rate is close to 0.5 per 1000 live births.

The early-onset form of GBS disease typically occurs in the first 24 hours of life, with fulminant sepsis or pneumonia and less often with meningitis. Despite intensive supportive care, diagnostic and

Correspondence to: L. Mahieu, University Hospital Antwerp Wilrijkstraat 10, BE 2650 Edegem, Belgium. Email: ludo.mahieu@uza.be therapeutic progresses, these infections have remained associated to high mortality (5-20\%) and morbidity; more than $30 \%$ of infants recovering of meningitis develop long-term neurological sequelae. ${ }^{5}$

In perinatal infections or EOD, GBS is transmitted vertically to the newborn from the vagina of a typically asymptomatic colonized woman during labour and delivery. In addition to colonization with GBS, other factors increase the risk for GBS EOD. These include prematurity (gestation $<37$ weeks), intrapartum fever (temperature $\geq 38^{\circ} \mathrm{C}$ ), duration of amniotic membrane rupture $\geq 18$ hours, previous delivery of an infant with invasive GBS disease, and GBS bacteriuria during current pregnancy. ${ }^{6,7}$

Because of the continuing magnitude and severity of GBS disease, several preventive strategies have been evaluated. ${ }^{8,9}$ The reference recommendations were published by the CDC in 1996, re-evaluated and 
updated 2002 and further in $2010 .{ }^{10}$ Universal screening at 35-37 weeks' gestation for maternal GBS colonization and use of intrapartum antibioprophylaxis is currently considered to be the most effective strategy to decrease the incidence of EOD. However, the cost of prenatal screening and antibiotic prophylaxis, as well as the selective pressure that antibiotics may have on the bacterial flora of mother and newborn, still generates much controversy.

In Belgium, as in many European countries, national guidelines for the GBS EOD prevention are currently available since 2003. ${ }^{11}$ Indeed, most hospitals have implemented strategies to decrease perinatal GBS infections and obstetric programs already included a GBS prevention policy but not always according to CDC guidelines. ${ }^{12} \mathrm{As}$ in the USA, despite the Belgian implemented strategy for prevention of perinatal GBS disease, GBS remains an important cause of GBS EOD.

The majority (95\%) of children with GBS EOD will become ill within the first 24 hours after birth. The management of ill neonates and/or neonates at high risk of infection is well defined, but management of healthy-appearing neonates is more problematic. An updated approach for empirical management of infants born to women, who received or should have received intrapartum antibiotic prophylaxis (IAP) to prevent GBS EOD or to treat suspected chorioamnionitis, is provided (Fig. 1) and was adapted from international guidelines. ${ }^{10,13}$ Indeed, both prenatal exposure and postnatal prolonged therapy with antibiotics has been associated with the development of necrotizing enterocolitis. ${ }^{14-17}$ The main objective of developing an algorithm for management of newborns is to minimize unnecessary evaluations and antimicrobial treatment in infants whose mothers received IAP.

\section{Methods}

This report was developed by a national GBS working group of paediatricians of the Belgian Superior Health Council in collaboration with gynaecologists and microbiologist according to the best available evidence (Table 1) and on experts' opinion when inadequate evidence was present. ${ }^{18}$ It represents the core of the paediatric section of the updated Belgian guidelines for prevention of perinatal GBS disease (not yet published). The level of evidence is presented between brackets throughout the guideline. The guidelines have been endorsed by the Belgian, Flemish, and French speaking sections of neonatology and paediatrics.

\section{Management of Neonate at Risk for Early-Onset GBS EOD}

Neonates with signs of neonatal sepsis

In any infant with clinical signs of sepsis, a full diagnostic evaluation should be carried out and empirical antibiotic therapy (ampicillin/amoxicillin or penicillin + aminoglycoside) should be started regardless of IAP, other obstetrical risk factors or, maternal GBS status (AII).

Because of sub-optimal sensitivity and specificity, and of poor predictive value for infection, routine use of urine antigen, cultures of mucous membranes, and gastric aspirates or body surfaces are not recommended. ${ }^{19-22}$

- clinical signs of sepsis: infant with a combination of signs as respiratory (apnea, grunting, tachypnea, cyanosis), cardiovascular (reduced capillary refill, hypotension, shock), central nervous system (lethargy, hypothermia, fever, seizures, apnoeic spells, irritability, bulging fontanel), or gastrointestinal (poor feeding, abdominal distension) disturbances;

- full diagnostic evaluation: full blood cell count (FBC) and differential, C-reactive protein (CRP) level, blood culture, lumbar puncture if feasible [cerebrospinal fluid (CSF) analysis and culture], chest X-ray, endotracheal culture (in intubated infants or if respiratory distress or lung infiltrate) to guide antibiotic treatment in blood culture negative 'pneumonia' cases. ${ }^{23}$

A lumbar puncture is indicated in all neonates with high suspicion of GBS infection especially in those with clinical signs of meningeal inflammation (seizures, apnoeic spells, irritability, bulging fontanel), in those who initially worsen with antimicrobial therapy, and all neonates with positive blood cultures. Indeed, $15 \%$ of infants with early-onset GBS infection have meningitis and $20 \%$ of infants with proven GBS meningitis have a negative blood culture. ${ }^{24,25}$ In cases of clinical instability, antibiotic therapy should be administered and lumbar puncture should be deferred and performed later until 48 hours for cell count, chemistry together with a blood glucose level for comparison and culture. An elevated CSF protein is the most sensitive parameter for GBS meningitis and a low CSF glucose is the most specific. Normal value depends on gestational age (Table 2). Of the infants with GBS meningitis $96 \%$ will have at least one abnormal CSF value. If meningitis is diagnosed, the dose of penicillin should be doubled and the duration of therapy extended to 2 weeks in GBS meningitis. Aminoglycosides should be stopped as soon as blood culture confirms GBS infection.

\section{Healthy-appearing newborn}

\section{Neonates at high risk for early-onset sepsis}

Neonates born to mothers with clinical chorioamnionitis predispose to infection with Gram-negative organisms and increase the risk of GBS infection in GBS colonized women. If a mother received intrapartum antibiotics for treatment of suspected chorioamnionitis, a limited diagnostic evaluation consisting of blood culture, FBC [including white blood cell (WBC) differential] + CRP at 12 hours and at 36 hours should be carried out; no chest radiograph or lumbar puncture 


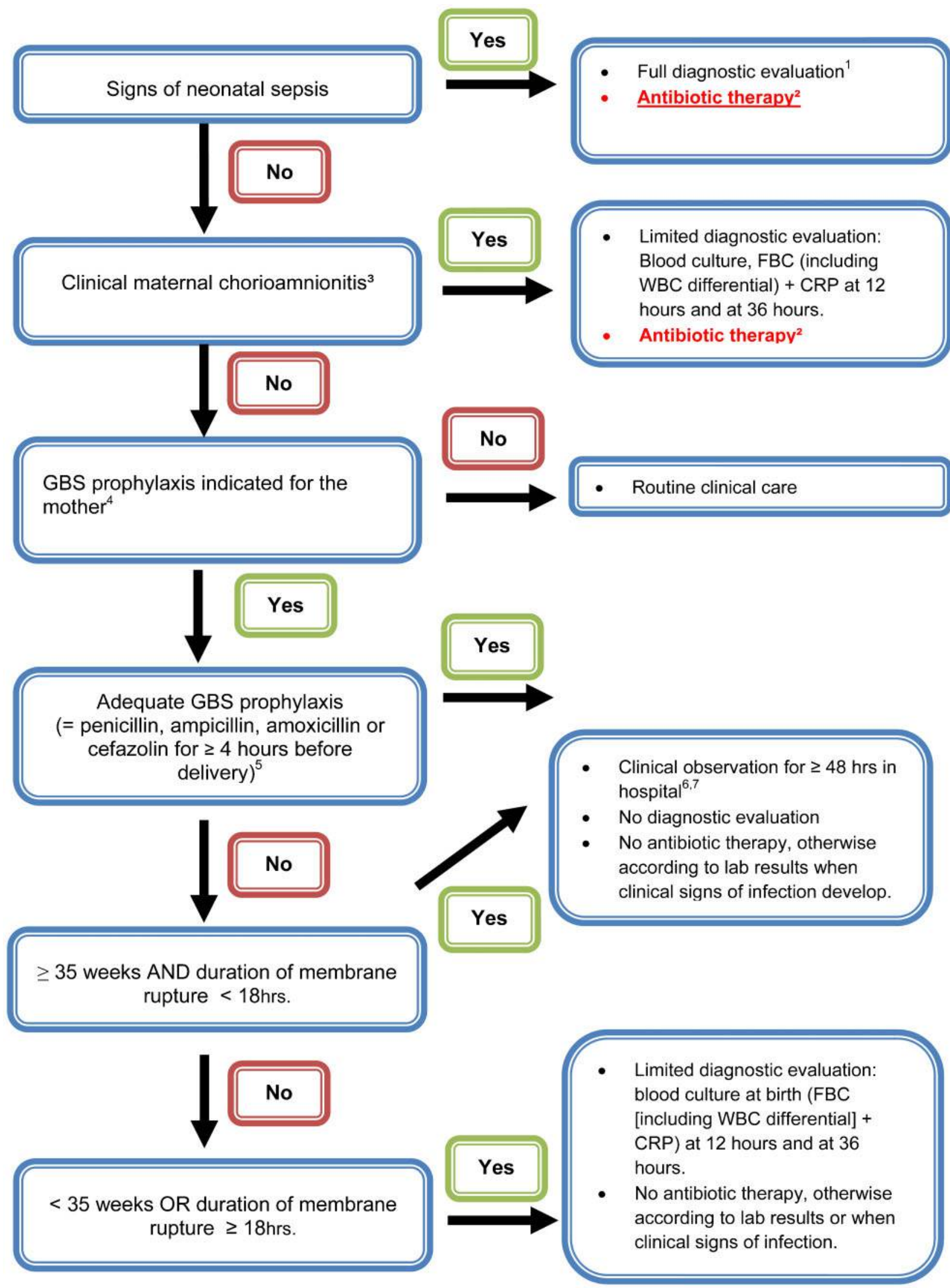

Figure 1 Algorithm for secondary prevention of early-onset GBS disease among newborns. ${ }^{1}$ Full diagnostic evaluation: blood culture, an FBC including white blood cell differential and platelet counts, CRP, chest X-ray (if respiratory symptoms are present), and lumbar puncture (at least if central nervous system signs are present, blood culture becomes positive and patient is stable enough to tolerate the procedure). Normal CSF values if $<37$ weeks gestation: WBC $<0.026 \times 10^{9} /$, glucose $>1.27 \mathrm{mmol} / \mathrm{l}$, protein $<15.1 \mathrm{~g} / \mathrm{l}$; if $\geq 37$ weeks gestation: WBC $<0.023 \times 10^{9} / \mathrm{l}$, glucose $>1.83 \mathrm{mmol} / \mathrm{l}$, protein $<17.1 \mathrm{~g} / \mathrm{l}^{2}$ Antibiotic therapy: ampicillin or Penicillin IV: double dose in case of meningitis or severe GBS sepsis. Aminoglycoside IV: measure serum concentration when treating for more than 48 hours. ${ }^{3}$ Consultation with obstetrician for clinical signs of 
chorioamnionitis (e.g. maternal fever $>38^{\circ} \mathrm{C}$, uterine tenderness, leukocytosis $>15 \times 10^{9} / \mathrm{l}$, foul smelling amniotic fluid, and/or fetal tachycardia) is important. Beware for intrapartum fever due to epidural anaesthesia. ${ }^{4}$ Indication for intrapartum GBS antibiotic prophylaxis: (1) previous infant with GBS disease; (2) GBS bacteriuria during this pregnancy; (3) GBS vagino-rectal culture positive during current pregnancy (35-37 weeks) or intrapartum nucleic acid amplification test (NAAT) positive for GBS on vaginal specimen: unless a cesarean delivery, is performed before onset of labor on a woman with intact amniotic membranes; (4) unknown GBS status at the onset of labor and $\geq 1$ risk factor at onset of labor: $<37$ weeks of gestation; amniotic membrane rupture $\geq 18$ hours; intrapartum temperature $\geq 38.0^{\circ} \mathrm{C}$; intrapartum nucleic acid amplification test (NAAT) positive for GBS. ${ }^{5}$ The efficacy of other antibiotics (e.g. vancomycin, clindamycin) has not been studied and, for this reason, from the paediatric management point of view, they are considered as 'inadequate' to protect the child against GBS infection. All oral antibiotics (e.g. azithromycin) should be considered as inadequate for GBS prophylaxis. ${ }^{6}$ Patient can be discharged home as early as $\mathbf{2 4}$ hours after delivery if $\geq 37$ weeks of gestation, assuming that other discharge criteria have been met, ready access to medical care exist, and a person is able to comply fully with instruction for home observation (e.g. midwife, generalist) and an infection is excluded by FBC+CRP after 24 hours. ${ }^{7}$ If signs of sepsis develop, a full diagnostic evaluation should be conducted and antibiotic therapy initiated.

is needed. Empiric antibiotic therapy (ampicillin/ amoxicillin or penicillin + aminoglycoside) should be started in these newborns regardless of clinical condition at birth or other conditions (AII). The term 'chorioamnionitis' is a clinical connotation of maternal fever $\left(>38^{\circ} \mathrm{C}\right)$ during labor (with or without uterine tenderness), leukocytosis, foul smelling amniotic fluid, and/or fetal tachycardia (CIII).

\section{Neonates at low risk of early-onset sepsis}

Routine use of antimicrobial prophylaxis and routine additional laboratory testing, as defined in this document is not recommended for healthy-appearing newborns whose mother received IAP. An algorithm for the management of these newborns is suggested in Fig. 1.

If no IAP was indicated, then no further diagnostic investigations or clinical observations are required (CIII).

If the mother received adequate IAP, which means penicillin, ampicillin, or cefazolin at least 4 hours before delivery, without additional risk factors
(PROM, preterm birth, and/or clinical chorioamnionitis), infants should be observed clinically in the mother's room without additional laboratory testing. When the newborn remains healthy, he or she can be discharged from the hospital after 48 hours. In case the neonate is $\geq 37$ weeks, gestation observation can occur at home, already after 24 hours, only when a person who is able to comply fully with instructions for home observation and access to medical care is readily available and a $\mathrm{FBC}+\mathrm{CRP}$ is negative after 24 hours. ${ }^{26}$ Agents other than penicillin, ampicillin, and cefazolin, such as clindamycin and vancomycin, although used in penicillin-allergic patients with a history of anaphylactic reaction, have not been proven efficient for IAP and are considered inadequate for purposes of IAP (CIII). Indeed, data on the ability of clindamycin and vancomycin to reach bactericidal levels in the fetal circulation and amniotic fluid are very limited and available data suggest that clindamycin provided to pregnant women do not reach fetal tissues reliably. ${ }^{27-31}$

Table 1 Evidence-based rating system used to determine strength of recommendations

\begin{tabular}{|c|c|c|}
\hline Category definition & & Recommended \\
\hline \multicolumn{3}{|l|}{ Strength } \\
\hline A & $\begin{array}{l}\text { Strong evidence for efficacy and substantial } \\
\text { clinical benefit }\end{array}$ & Strongly \\
\hline B & $\begin{array}{l}\text { Strong or moderate evidence for efficacy but } \\
\text { only limited clinical benefit }\end{array}$ & Generally \\
\hline C & $\begin{array}{l}\text { Insufficient evidence for efficacy or efficacy does } \\
\text { not outweigh possible adverse consequences }\end{array}$ & Optional \\
\hline $\mathrm{D}$ & Moderate evidence against efficacy or adverse outcome & Generally not \\
\hline $\mathrm{E}$ & Strong evidence against efficacy or adverse outcome & Never \\
\hline \multicolumn{3}{|l|}{ Quality of evidence } \\
\hline I & $\begin{array}{l}\text { Evidence from at least one well-executed randomized, } \\
\text { controlled trial or one rigorously designed laboratory-based } \\
\text { experimental study that has been replicated by an } \\
\text { independent investigator }\end{array}$ & \\
\hline II & $\begin{array}{l}\text { Evidence from at least one well-designed clinical trial without } \\
\text { randomization, cohort or case-controlled analytic studies } \\
\text { (preferably from more than one center), multiple time-series } \\
\text { studies, dramatic results from uncontrolled studies, or some } \\
\text { evidence from laboratory experiments }\end{array}$ & \\
\hline III & $\begin{array}{l}\text { Evidence from opinions of respected authorities based on } \\
\text { clinical or laboratory experience, descriptive studies, or } \\
\text { reports of expert committees }\end{array}$ & \\
\hline
\end{tabular}

Source: Adapted from La Force FM. ${ }^{18}$ 
Table 2 Normal cerebrospinal fluid parameters in neonates

\begin{tabular}{llll}
\hline Gestational age (weeks) & White blood cell count $(\times \mathbf{1 0} / \mathbf{l})$ & Glucose* $(\mathbf{m m o l} / \mathbf{l})$ & Protein $(\mathbf{g} / \mathbf{l})$ \\
\hline$<37$ & $<0.026$ & $>1.27$ & $<15.1$ \\
$\geq 37$ & $<0.023$ & $>1.83$ & $<17.1$ \\
\hline
\end{tabular}

Note: *Glycorachia should be $>75 \%$ of serum glycaemia.

Furthermore, in Belgium, a high resistance level $(>25 \%)$ of the GBS strains to clindamycin is reported by the national reference centre for GBS.

If no adequate maternal IAP for GBS has been administered, despite indication being present (e.g. other antibiotics such as penicillin, ampicillin and cefazolin for IAP, IAP $<4$ hours before delivery, no IAP despite indication), then algorithm of Fig. 1 is followed.

In case the infant is $\geq 35$ weeks and duration of membrane rupture was $<18$ hours, then the infant should be observed for at least 48 hours, and no routine diagnostic testing needs to be performed. If clinical signs of infection develop, a full diagnostic evaluation should be performed and empirical antibiotic therapy (ampicillin/amoxicillin + aminoglycoside) should be started (see the section on 'Neonates at high risk for early onset sepsis') (BIII).

In case the infant is $<35$ weeks or duration of membrane rupture was $\geq 18$ hours, then the infant should be observed for at least 48 hours, and limited diagnostic testing should be performed. Again, if clinical signs of infection develop, a full diagnostic evaluation, including cultures, should be undertaken and empirical antibiotic therapy (ampicillin/amoxicillin + aminoglycoside) should be started (the section on 'Neonates at high risk for early onset sepsis') (BIII).

\section{Which and When Laboratory Testing? Limited evaluation}

Limited evaluation includes blood culture (at birth), FBC with differential and platelets (at 6-12 hours of life), and CRP at 12 and 36 hours.

Because of the low sensitivity and specificity at birth, results of the FBC can provide more information about the presence of infection if the test is not performed before 6-12 hours after delivery. ${ }^{32,33}$ Clinical signs are much more sensitive than any laboratory test of infection. Therefore, treatment should rather be based on clinical signs and on maternal risk factors, (e.g. in the case of inadequate IAP: gestational age and PROM). Population data reveal that late preterm infants (35-36 weeks) are at low risk for GBS disease and related mortality compared to their more premature counterparts and even term infants. ${ }^{34}$ Therefore, in the algorithm, the cutoff was set at $<35$ weeks and not at 37 weeks as the definition for prematurity. Thus, laboratory tests should rather be seen as a confirmation of clinical judgment (e.g. positive laboratory tests in ill infants and negative tests in healthy-appearing neonates). In order to increase the diagnostic performance, serial measurements of CRP and FBC should be undertaken at least at around 12 and 36 hours of life. ${ }^{35}$

Lower and upper limits of neutrophils count vary with postnatal age. The total and differential WBC count are affected by several factors besides infection, including infant age in hours (lower first hours), the method of blood sampling (lower via arterial blood sampling), the method of delivery (lower after caesarean section), maternal hypertension (lower), infant's gender (lower in boys), and infant's gestational age (lower in very low birth weight infants and premies). ${ }^{36-38}$ Sepsis should be suspected if leukopenia $<5000 / \mathrm{mm}^{3}$, neutrophilia $>25 \times 10^{9} / 1$, leukocytosis $>30 \times 10^{9} / 1$ immature to total neutrophil count ( $\mathrm{I} / \mathrm{T}$ ratio $\geq 0.30$ ) or if neutropenia defined as absolute neutrophil count $<10$ th percentile adjusted for gestational age (Schmutz criteria) (Table 3). ${ }^{39}$ Thrombocytopenia is not a sign of sepsis within 24 hours after birth. ${ }^{32}$

The sensitivity of CRP) to predict a bacterial infection increases rapidly after birth, but at least 612 hours after the onset of infection are necessary to reach abnormal level. Therefore, if blood for CRP is taken for decision- making regarding initiating antimicrobial therapy, then the drawing can better be delayed for a few hours. A significant increase (CRP level above $10 \mathrm{mg} / \mathrm{l}$ ) between two serial measurements on samples taken over the first 848 hours of life has a sensitivity of almost $100 \%$ for an infectious status and normal levels for the two samples have a negative predictive value of $90-100 \%$ for an infectious status. ${ }^{40}$ The normal upper level of CRP depends on the laboratory, but in general, a CRP $>10 \mathrm{mg} / 1$ is considered a positive level. In premature infants, the increase of CRP may be delayed and the level may be lower because of immaturity of the liver. ${ }^{41}$

Sepsis should be suspected based on repeated clinical and laboratory evaluations and if sepsis is

Table 3 Criteria for lower limits of neutrophils $/ \mathrm{mm}^{3}$ at 68 hours after birth according to gestation (criteria according to Schmutz et al. ${ }^{32}$ )

\begin{tabular}{llll}
\hline Gestation & $<\mathbf{2 8}$ weeks & 28-36 weeks & $>\mathbf{3 6}$ weeks \\
\hline $\begin{array}{l}\text { Neutrophil } \\
\text { count }\left(\times 10^{9} / \mathrm{l}\right)\end{array}$ & 1.5 & 3.5 & 7.5 \\
\hline
\end{tabular}


suspected, a full diagnostic evaluation should be carried out (see the section on 'Neonates at high risk for early-onset sepsis'), including cultures, and empiric antibiotic therapy should be started.

\section{Empiric Antibiotic Therapy}

Ill neonates are those at risk with abnormal laboratory tests, which should be treated with antimicrobial agents active against GBS, as well as other organisms that might cause neonatal sepsis (e.g. ampicillin or penicillin + aminoglycoside). Antimicrobial switch to third-generation cephalosporin (e.g. cefotaxime) is necessary in case of Gram-negative sepsis/meningitis because of increasing ampicillin resistance of Escherichia coli. Dosage and regimen of antimicrobial agents depend on diagnosis, post-natal age, and birth weight. For dosages, we refer to the Sanford guide. ${ }^{42}$ Intravenous immunoglobulins do not improve morbidity nor mortality and are not indicated. ${ }^{43}$

Duration of antibiotic therapy varies depending on results of cultures and on the clinical course of the infant (Table 4): If GBS infection is confirmed by culture and meningitis is excluded, ampicillin should be replaced by the narrower spectrum penicillin and aminoglycoside should be discontinued. In the case of GBS meningitis, the dose of penicillin should be doubled. Combination therapy with aminoglycoside can be discontinued if CSF specimen obtained 48 hours in therapy is sterile. ${ }^{44,45}$ Ventriculitis is a common complication of neonatal meningitis. There are no reliable clinical signs of ventriculitis, although evidence of increased intracranial pressure usually is present. It must be suspected on the basis of failure to respond clinically and bacteriologically to appropriate antimicrobial therapy; if ventriculitis results in obstruction to CSF flow, the access of systemic antibiotics to the ventricular CSF can be limited. Neuroimaging should be performed to make the diagnosis. Cranial sonography can demonstrate findings suggestive of ventriculitis or obstructed flow of CSF. Contrast-enhanced computer tomography or magnetic resonance imaging can demonstrate enhancement of the lining of the ventricles. Ventricular fluid aspiration is indicated for infants who have ventriculitis with an obstruction to the flow of CSF. In this setting, cultures of CSF often remain positive for the infecting organism for several days or longer. Treatment can involve direct instillation of an antimicrobial such as gentamicin or amikacin directly into the ventricle. The duration of antimicrobial therapy may extend several weeks longer than the time required to sterilize the ventricular $\mathrm{CSF}$ and can be as long as $6-8$ weeks.

\section{Future Perspectives}

A major change in the new guideline is that asymptomatic term 'neonates' should not have any laboratory testing unless any additional risk factors such as PROM even when GBS prophylaxis was inadequate. This will decrease unnecessary evaluations and antibiotic exposure in healthy neonates. National long-term surveillance of early-onset infections in both preterm and term infants remains of high value. Not only is this important to monitor the effect of intrapartum prophylaxis on the incidence of GBS disease, but also to detect emergence resistance of GBS isolates and to find emerging neonatal pathogens causing early-onset sepsis. If there is no GBS vaccine available, universal screening and intrapartum antimicrobial prophylaxis is the best option for the prevention of neonatal GBS disease and mortality. Future research should focus on the value of rapid molecular testing in order to identify not only colonized mothers, but also children at highest risk of invasive disease.

\section{Disclaimer Statements}

\section{Contributors None.}

\section{Funding None.}

Conflicts of interest The undersigned authors declare that there is no affiliation, financial agreement, or other involvement with any company and no other conflicts of interest.

\section{Ethics approval None.}

\section{Acknowledgements}

The authors extend their sincere thanks to the other Belgian Health Council GBS working group members [S. Alexander (ULB, Brussels), A. Naessens (UZB, Brussels), C. Potvliege (CHU, Tivoli), G. Claeys (University Hospital Ghent), G. Donders (University Hospitals Leuven), C. Hubinont (Catholic University Louvain-la-Neuve), K. Roelens, (University Hospital Ghent), M. Temmerman (University Hospital Ghent), W. Foulon (University Hospital Brussels), A. Vanholsbeeck (University Hospital Ghent)]. They would also like to thank the chairman of the local scientific

Table 4 Duration of antibiotic therapy according to focus of infection and culture results

Focus of infection

Suspected sepsis not confirmed by clinical, biological or bacteriological results Proven sepsis

Meningitis

Ventriculitis (*)

Osteomyelitis

\section{Duration of therapy}

36-48 hours

7-10 days

14 days minimum

28 days

4-6 weeks 
paediatric committee (M. Raes) and the presidents of the scientific regional committees of neonatology (G. Naulaers and P. Maton) for their critical revision of the guidelines.

\section{References}

1 Baker CJ, Barrett FF. Transmission of group B streptococci among parturient women and their neonates. 1973;83:919-25.

2 Boyer KM, Gotoff SP. Prevention of early-onset neonatal disease with selective intrapartum chemoprophylaxis. N Engl J Med. 1986;314:1665-9.

3 Davies HD, Raj S, Adair C, Robinson J, McGeer A; Alberta GBS Study Group. Population-based active surveillance for neonatal group B streptococcal infections in Alberta, Canada: implications for vaccine formulation. Pediatr Infect Dis J. 2001;20:879-94.

4 Trijbels-Smeulders M, de Jonge GA, Pasker-de Jong PC, Gerards LJ, Adriaanse AH, vanLingen RA, et al. Epidemiology of neonatal group $\mathrm{B}$ streptococcal disease in the Netherlands before and after introduction of guidelines for prevention. Arch Dis Child Fetal Neonatal Ed. 2007;92(4):F271-6.

5 Embleton N, Wariyar U, Hey E. Mortality from early-onset group B streptococcal infection in the United Kingdom. Arch Dis Child Fetal Neonatal Ed. 1999;80:F139-41.

6 Schuchat A, Wenger JD. Epidemiology of group B streptococcal disease: risk factors, prevention strategies, and vaccine development. Epidemiol Rev. 1994;16:374 402.

7 Fargason C, Peralta-Carcelen M, Rouse D, Cutter G, Goldenberg R. The pediatric cost of strategies for minimizing the risk of early-onset group B streptococcal disease. Obstet Gynecol. 1997;90:347-52.

8 Hager WD, Schuchat A, Gibbs R, Sweet R, Mead P, Larsen JW. Prevention of perinatal group B streptococcal infection: current controversies. Obstet Gynecol. 2000;96:141-5.

9 Schrag SJ, Zell ER, Lynsfield R, Roome A, Arnold KE, Craig AS, et al. A population-based comparison of strategies to prevent early-onset group B streptococcal disease in neonates. N Engl J Med. 2002;347:233-9.

10 Verani JR, McGee L, Schrag SJ; Division of Bacterial Diseases, National Center for Immunization and Respiratory Diseases, Centers for Disease Control andPrevention (CDC). Prevention of perinatal group B streptococcal disease - revised guidelines from CDC, 2010. MMWR Recomm Rep. 2010;59(RR-10):1-36.

11 Melin P, Verschraegen G, Mahieu L, Claeys G, Mol PD. Towards a Belgian consensus for prevention of perinatal group B streptococcal disease. Indian J Med Res. 2004;119(Suppl): 197-200.

12 Mahieu L, de Dooy J, Leys E. Obstetricians' compliance with CDC guidelines on maternal screening and intrapartum prophylaxis for group B streptococcus. J Obstet Gynecol. 2000;20:460-4.

13 Polin RA, Committee on Fetus and Newborn. Management of neonates with suspected or proven early-onset bacterial sepsis. Pediatrics. 2012;129:1006-15.

14 Alexander VN, Northrup V, Bizzarro MJ. Antibiotic exposure in the newborn intensive care unit and the risk of necrotizing enterocolitis. J Pediatr. 2011;159(3):392-7.

15 Kuppala VS, Meinzen-Derr J, Morrow AL, Schibler KR. Prolonged initial empirical antibiotic treatment is associated with adverse outcomes in premature infants. J Pediatr. 2011; 159(5):720-5.

16 Abdel Ghany EA, Ali AA. Empirical antibiotic treatment and the risk of necrotizing enterocolitis and death in very low birth weight neonates. Ann Saudi Med. 2012;32(5):521-6.

17 Weintraub AS, Ferrara L, Deluca L, Moshier E, Green RS, Oakman E, et al. Antenatal antibiotic exposure in preterm infants with necrotizing enterocolitis. J Perinatol. 2012;32(9): 705-9.

18 LaForce FM. Immunizations, immunoprophylaxis, and chemoprophylaxis to prevent selected infections. US Preventive Services Task Force. JAMA. 1987;257(18):2464-70.

19 Perkins MD, Mirrett S, Reller LB. Rapid bacterial antigen detection is not clinically useful. J Clin Microbiol. 1995;33(6): 1486-91.

20 Williamson M, Fraser SH, Tilse M. Failure of the urinary group B streptococcal antigen test as a screen for neonatal sepsis. Arch Dis Child Fetal Neonatal Ed. 1995;73:F109-11.
21 Hall RT, Kurth CG. Value of negative nose and ear cultures in identifying high-risk infants without early-onset group B streptococcal sepsis. J Perinatol. 1995; 15:356-8.

22 Borderon E, Desroches A, Tescher M, Bondeux D, Chillou C, Borderon JC. Value of examination of the gastric aspirate for the diagnosis of neonatal infection. Biol Neonate. 1994;65(6):353-66.

23 Booth GR, Al-Hosni M, Ali A, Keenan WJ. The utility of tracheal aspirate cultures in the immediate neonatal period. $\mathbf{J}$ Perinatol. 2009;29:493-6.

24 Wiswell TE, Baumgart S, Gannon CM, Spitzer AR. No lumbar puncture in the evaluation for early neonatal sepsis: will meningitis be missed? Pediatrics. 1995;95:803-6.

25 Randis TM, Polin RA. Early-onset group B Streptoccal sepsis: new recommendations from the Centres for Disease Control and Prevention. Arch Dis Child Fetal Neonatal Ed. 2012;97:F291-4.

26 Philip AG. White blood cells and acute phase reactants in neonatal sepsis. Pediatrie. 1984;39:371-8.

27 Pacifici GM. Placental transfer of antibiotics administered to the mother: a review. Int J Clin Pharm Ther. 2006;44:57-63.

28 Laiprasert J, Klein K, Mueller BA, Pearlman MD. Transplacental passage of vancomycin in noninfected term pregnant women. Obstet Gynecol. 2007;109:1105-10.

29 Philipson A. Pharmacokinetics of antibiotics in pregnancy and labour. Clin Pharmacokinet. 1979;4:297-309.

30 Philipson A, Sabath LD, Charles D. Transplacental passage of erythromycin and clindamycin. N Engl J Med. 1973;288:1219-21.

31 Muller A, Mouton J, Oostvogel P, Dörr PJ, Voskuyl RA, DeJongh $\mathrm{J}$, et al. Pharmacokinetics of clindamycin in pregnant women in the peripartum period. Antimicrob Agents Chemother. 2010;54:2175-81.

32 Newman TB, Puopola KM, Wi S, Draper D, Escobar GJ. Interpreting complete blood counts soon after birth in newborns at risk for sepsis. Pediatrics. 2011;126:903-9.

33 Ottolini M, Lundgren K, Mirkinson L, Cason S, Ottolini MG. Utility of a complete blood count and blood culture screening to diagnose neonatal sepsis in the asymptomatic newborn. Pediatr Infect Dis J. 2003;22:430-4.

34 Stoll BJ, Hansen NI, Sanchez PJ, Faix RG, Poindexter BB, Van Meurs KP, et al. Early onset neonatal sepsis: the burden of group B streptococcal and E. coli disease continues. Pediatrics. 2011;127:817-26.

35 Schouten-van Meeteren NY, Rietveld A, Moolenaar AJ, van Bel F. Influence of perinatal conditions on $\mathrm{C}$-reactive protein production. J Pediatr. 1992;120:621-4.

36 Manroe BL, Rosenfeld CR, Weinberg AG, Browne R. The differential leukocyte count in the assessment and outcome of early-onset neonatal group B streptococcal disease. J Pediatr. 1977;91:632-7.

37 Mouzinho A, Rosenfeld CR, Sánchez PJ, Risser R. Revised reference ranges for circulating neutrophils in very-low-birthweight neonates. Pediatrics. 1994;94:76-82.

38 Escobar GJ, Li D, Armstrong MA, Gardner MN, Folck BF, Verdi JE, et al. Neonatal sepsis workups in infants $\geq 2000$ grams at birth: a population-based study. Pediatrics. 2000;106:256-63.

39 Schmutz N, Henry E, Jopling J, Christensen RD. Expected ranges for blood neutropphil concentrations of neonates: the Manrou and Mouzinho charts revisited. J Perinatol. 2008;28(4): 275-81.

40 Benitz WE, Han My, Madan A, Ramachandra P. Serial serum C-reactive protein levels in the diagnosis of neonatal infection. Pediatrics. 1998;102:E41.

41 Zukowsky K, Greenspan JS. Beyond the basics: advanced physiology and care concepts. Adv Neonatal Care. 2003;3(1):3-13.

42 Independent Belgian/Luxembourg Working Party on Antimicrobial Therapy. The Sanford Guide to Antimicrobial Therapy 23rd edition of the Belgian/Luxembourg Version 2012-2013 (adapted for use by the medical profession in Belgium and Luxembourg by the independent Belgian/ Luxembourg Working Party on Antimicrobial Therapy (distributed under license by the Société belge d'infectiologie et de microbiologie clinique BIMC/BVIKM, pp. 1-500). Sperryville, VA: Antimicrobial Therapy Inc.

43 INIS Collaborative Group, Brocklehurst P, Farrell B, King A, Juszczak E, Darlow B, Haque K, et al. Treatment of neonatal sepsis with intravenous immune globulin. $\mathrm{N}$ Engl J Med. 2011;365(13):1201-11.

44 NICE clinical guideline 149: antibiotics for early-onset neonatal infection 2012. London: NICE; 2012.

45 Remington JS, Klein JO. Infectious diseases of the fetus and newborn. 7th ed. Philadelphia, PA: Elsevier/Saunders; 2011. 